MICROBIOME

\title{
Silencing Staphylococcus aureus with probiotics
}

B. subtilis

spores ...

significantly

reduced

S. aureus gut colonization when fed to mice

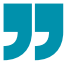

Probiotics are widely consumed and promoted as providing health benefits. Specifically, probiotic bacteria have been proposed to reduce colonization of the gut mucosa by pathogens and thus prevent infections. However, the use of probiotics is controversial as evidence of their colonization efficacy remains sparse and the underlying mechanisms that might provide health benefits are unknown. Moreover, there is limited evidence to indicate that probiotic bacteria directly interfere with pathogens. Now, Piewngam et al. report that the consumption of probiotic Bacillus bacteria abolishes Staphylococcus aureus gut colonization by interfering with $S$. aureus quorum-sensing.

The authors observed a lower rate of $S$. aureus gut colonization in a rural Thai population compared with individuals from urbanized Western areas, which they hypothesized could be due to bacterial interactions in the gut. To test this hypothesis, they surveyed the gut microbiome of 40 randomly selected individuals (20 S. aureus carriers and 20 non-carriers) and

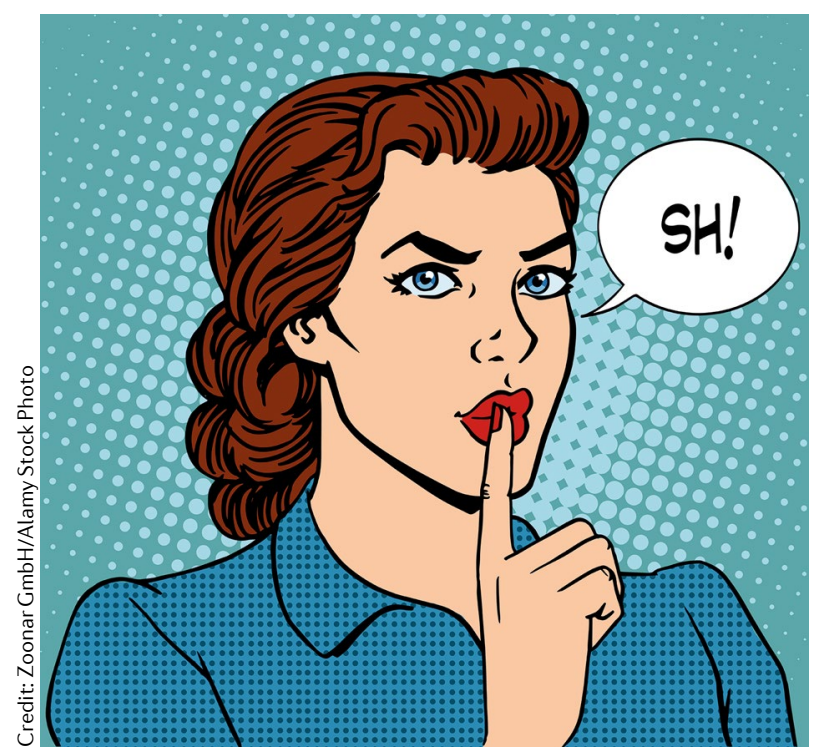

observed no substantial high-order taxonomic difference in the composition of the gut microbiome between $S$. aureus carriers and non-carriers. However, a strong correlation was observed between the absence of $S$. aureus and the presence of Bacillus spp. (mostly Bacillus subtilis). Using culture-based analysis, $S$. aureus was never detected when Bacillus spp. were present and the level of $S$. aureus colonization in non-Bacillus-colonized individuals was similar to Western individuals, which led the authors to hypothesize that Bacillus spp. produce a substance that interferes with $S$. aureus colonization.

Previous work suggested that the accessory gene regulator (Agr) quorum-sensing system could regulate S. aureus colonization, prompting the authors to investigate the relationship between Agr quorum-sensing and colonization, and whether Bacillus spp. secrete a substance that affects Agr quorum-sensing. In a mouse model of S. aureus intestinal colonization, competition experiments between wild-type S. aureus and isogenic agr mutants revealed that only wild-type bacteria colonized the gut, and only bacteria that expressed the intracellular Agr effector RNAIII could colonize. Therefore, the authors purport that Agr quorum-sensing is indispensable for S. aureus gut colonization.

Next, they assessed whether culture filtrates of Bacillus spp. isolates from human faeces could inhibit Agr quorum-sensing. Filtrates from all 105 isolates reduced Agr signalling in an $S$. aureus reporter strain, but did not inhibit cell growth, indicating that Bacillus spp. release a substance that inhibits Agr signalling. Using reversed-phase high-performance chromatography, the fraction containing the
Agr-inhibiting activity was isolated from the filtrate, which was subsequently analysed by mass spectrometry to identify members of the fengycin cyclic lipopeptide family as the molecules responsible for Agr inhibition.

Isolated $\beta$-OH-C17-fengycin B (the most abundant fengycin in filtrates) inhibited Agr signalling at concentrations observed in stationary-phase cultures of Bacillus isolates. Strong evidence that fengycins are the inhibitory molecules came from experiments in which the fenA gene (essential for fengycin synthesis) in B. subtilis was mutated; the fenA mutant was unable to synthesize fengycins and inhibit Agr activity, confirming that fengycins are responsible for the observed inhibition.

As fengycins are cyclic lipopeptides that show structural similarity to Agr autoinducing peptides (AIPs) that are part of the quorum-sensing regulatory circuit, the authors hypothesized that fengycins compete with AIPs for binding to the extracellular sensor kinase. In support of this, fengycin Agr inhibition was reversed in a dose-dependent manner by adding AIPs, and fengycin could inhibit Agr at AIP concentrations that are found during the early stationary growth phase, indicating that fengycins inhibit Agr signalling by competitive inhibition. Purified $\beta$-OH-C17-fengycin B also inhibited Agr signal transduction in members of all S. aureus subtypes and the related Staphylococcus epidermidis, suggesting that fengycins have broad-spectrum activity.

Importantly, wild-type $B$. subtilis spores - but not an isogenic fen $A$ mutant - significantly reduced S. aureus gut colonization when fed to mice, suggesting that Bacillus spp. could be used as a probiotic for $S$. aureus decolonization in humans.

Ashley York

ORIGINAL ARTICLE Piewngam, P. et al. Pathogen elimination by probiotic Bacillus via signaling interference. Nature https://doi.org/10.1038/ s41586-018-0616-y (2018) 